\title{
Roda de conversa no CECCO: uma experiência
}

\author{
Conversation circle at CECCO: an experience \\ Rueda de conversación en CECCO: una experiencia
}

Recebido: 03/03/2021 | Revisado: 07/03/2021 |Aceito: 11/03/2021 | Publicado: 19/03/2021

\author{
Maria Cecília Leite de Moraes \\ ORCID: https://orcid.org/0000-0002-8717-6513 \\ Universidade Federal da Bahia, Brasil \\ E-mail: leimo7@hotmail.com \\ Dálity Keffelen de Barros Rodrigues \\ ORCID: https://orcid.org/0000-0003-0835-0948 \\ Coordenadoria do Controle de Doenças de São Paulo, Brasil \\ E-mail: barrosbiomed@gmail.com
}

\begin{abstract}
Resumo
O câncer de mama entre as mulheres apresenta alta prevalência e, importante índice de mortalidade no Brasil. Diante do cenário, o Grupo PAPO DE SAÚDE e os servidores do CECCO organizaram uma RODA DE CONVERSA com o objetivo de dialogar sobre as ações primárias para combate da doença. A metodologia que compõe essa formatação é a pesquisa-ação comunitária. Os convites foram feitos e as presenças foram espontâneas. Optou-se por não identificar e numerar as presentes devido as características do serviço. Os resultados foram positivos evidenciando o conhecimento do tema entre as mulheres que participaram da atividade. A avaliação pontuou o êxito da iniciativa e a perspectiva de futuras RODAS DE CONVERSA, que poderão se consolidar como uma atividade mais frequente entre CECCO e PAPO DE SAÚDE.
\end{abstract}

Palavras-chave: Câncer de mama; Roda de conversa; Promoção de saúde.

\begin{abstract}
Breast cancer among women has a high prevalence and an important mortality rate in Brazil. In view of the scenario, the PAPO DE SAÚDE Group and the CECCO employees organized a CONVERSATION WHEEL with the objective of talking about the primary actions to combat the disease. The methodology that makes up this format is community action research. The invitations were made and the attendance was spontaneous. It was decided not to identify and number those present due to the characteristics of the service. The results were positive, showing the knowledge of the theme among the women who participated in the activity. The evaluation highlighted the success of the initiative and the perspective of future CONVERSATION WHEELS, which could be consolidated as a more frequent activity between CECCO and PAPO DE SAÚDE.
\end{abstract}

Keywords: Breast cancer; Conversation wheel; Health promotion.

\section{Resumen}

El cáncer de mama entre las mujeres tiene una alta prevalencia y una importante tasa de mortalidad en Brasil. Ante el escenario, el Grupo PAPO DE SAÚDE y los empleados de CECCO organizaron una RUEDA DE CONVERSACIÓN con el objetivo de hablar sobre las principales acciones para combatir la enfermedad. La metodología que compone este formato es la investigación-acción comunitaria. Las invitaciones se hicieron y la asistencia fue espontánea. Se decidió no identificar y numerar a los presentes por las características del servicio. Los resultados fueron positivos, mostrando el conocimiento del tema entre las mujeres que participaron en la actividad. La evaluación destacó el éxito de la iniciativa y la perspectiva de futuras RUEDAS DE CONVERSACIÓN, que podrían consolidarse como una actividad más frecuente entre CECCO y PAPO DE SAÚDE.

Palabras clave: Cáncer de mama; Rueda de conversación; Promoción de la salud.

\section{Introdução}

$\mathrm{O}$ autocuidado refere-se à responsabilidade das pessoas com relação a sua própria saúde, permitindo que indivíduos cuidem de si, com pouca ou nenhuma ajuda. São ações conscientes, que beneficiam a própria pessoa, sendo um vigoroso aliado para a prevenção de doenças e preservação da vida (Silva \& Domingues, 2017). Sabe-se que, no Brasil, apesar da pujança do Sistema Único de Saúde (SUS), muitos encontram-se desassistidos, principalmente pela imensidão do país e o enorme número de habitantes (Maropo, Santana, Oliveira Filho, Almeida, \& Araújo, 2015). Diante disso, a educação popular em saúde, 
formulada com a contribuição da academia e servidores da saúde, possui um papel fundamental na informação de populações (Raimondi et al., 2018). Trabalhar com grupos vulneráveis pode trazer mudanças expressivas. Tais premissas oportunizaram a elaboração de uma RODA DE CONVERSA com mulheres frequentadoras de um CECCO voltado para a prevenção e combate ao câncer de mama.

\section{O Brasil e a Saúde da Mulher}

Inicialmente, torna-se importante mencionar que o termo gênero, muitas vezes, é utilizado incorretamente, focado apenas em aspectos biológicos, marcando um constructo referente a padrões de condutas (Barata, 2009). No Brasil, a saúde integral da mulher tornou-se um tema relevante no início do século 20. Até então, os cuidados estavam limitados ao período da gravidez e do parto, entendendo-se que o ciclo de vida feminino estava ligado a reprodução, cuidados maternos e supervisão familiar (Tavares et al., 2020). Diante desta perspectiva, que alijava a mulher de seus múltiplos lugares, institui-se o Programa de Assistência à Saúde da Mulher (PAISM), formulado pelo Ministério da Saúde no ano de 1983. Apresenta como seu eixo norteador a compreensão da mulher a partir de um contexto sociocultural com sua gama de necessidades. Valoriza o vínculo com os serviços (Martins et al., 2018), tornando-se uma referência nas questões da saúde feminina, incorporando, além dos cuidados próprios de gênero, o planejamento familiar e os direitos sexuais, assuntos debatidos anteriormente em conferências internacionais do Cairo e Beijing. O programa contempla a saúde da mulher considerando todo seu ciclo de vida (Bankoff, 2014). Em suma, o PAISM é o suporte de cuidados voltado para essa população (Oliveira, 2018).

\section{O Câncer de Mama}

No Brasil, o câncer de mama apresenta uma alta incidência, sendo o segundo em número de casos entre as mulheres, ficando apenas atrás do câncer de pele não melanoma (Rodrigues et al. 2019). É um importante problema de saúde pública no que diz respeito também aos seus índices de mortalidade (Ayala, Anjos, Cassol, Höfelmann, 2019). Enfatiza-se, ainda, o aumento do número de casos em diversas regiões do mundo, sendo que o diagnóstico tem ocorrido em estágios bastante avançados (Alves, Ferreira, Santos, Almeida, Fernandes, 2019). É importante mencionar que no período entre 2020-2022 os números de casos estimados para o Brasil alcançarão 66.280 casos por ano (Costa et al., 2020). Dentre os principais fatores de risco para a presença da doença está o histórico familiar e seus indicadores genéticos, menarca precoce, menopausa tardia, sedentarismo, obesidade, nuliparidade, existência de vícios e idade avançada (Rodrigues, Cruz, Paixão, 2015). As ações preventivas são fundamentais para a mudança de cenário e estão divididas em primárias e secundárias. Distinguem-se como ações primárias: atividade física, alimentação saudável, amamentação, ingestão equilibrada de bebidas alcoólicas e controle de peso (Viegas et al., 2019). As secundárias referem-se as ações específicas para o combate ao câncer de mama, como medidas de diagnóstico precoce, destacando-se os exames para detecção da doença (Costa et al., 2020).

\section{O CECCO}

Os Centros de Convivência e Cooperativa (CECCO’s) são dispositivos criados para compor com a Reforma Psiquiátrica e adjuvar a Atenção Psicossocial (Aleixo \& Lima, 2017). Subsidiam as mudanças presentes nas propostas de um novo modelo de cuidado na saúde mental, transformando-se em importantes alternativas aos hospitais psiquiátricos e, padrões de assistência para as pessoas usuárias destes equipamentos (Alvarez, Silva, \& Oliveira, 2016). Abre-se um rápido parêntesis para relatar a concepção de Reforma Sanitária. Tratou-se de um processo sociopolítico, que contou com a participação de instituições e atores presentes em diferentes esferas de poder e entidades de educação. $\mathrm{O}$ foco principal do movimento foi modificar o olhar, as práticas, os valores e os cuidados no que se refere aos pacientes portadores de transtorno mental. 
Envolveu, de maneira especial, aspectos da não hospitalização e da convivência rotineira desses indivíduos na família e na comunidade (Alvarez et al., 2016). Com este pano de fundo, os CECCO's estabeleceram-se, inicialmente nos anos 80, nas cidades de Belo Horizonte, Campinas e São Paulo (Aleixo \& Araújo Lima, 2017). Esta última configurou esses equipamentos, de maneira inovadora e ampla, substituindo as redes e serviços tradicionais (Silva, Barbosa, \& Bonini-Trenche, 2020). Emergiram lugares com vocação revolucionária focados no apoio de indivíduos em situação de vulnerabilidade donde se incluíram, também, os usuários de drogas (Aleixo \& Araújo Lima, 2017). Além do que, os serviços abraçaram pessoas que apresentavam questões contextuais, déficits econômicos e ambientais, que levavam à solidão e ao sofrimento. Instituíram-se como espaços transversais e interseccionais de circulação, inclusão, socialização e promoção da saúde (Caçapava, Colvero, Pereira, 2009). A Promoção da Saúde merece destaque neste contexto, já que na elaboração da teoria da História Natural da Doença, foi considerada uma tipologia de atenção à saúde que estabelecia o cuidar antes de adoecer. O refinamento da concepção traz como propósito precípuo contemplar ações que capacitam e responsabilizam indivíduos e grupos na preservação da saúde; aspecto que abrange qualidade de vida em um espectro extremamente amplo (Moraes, 2017). O CECCO é reconhecidamente um importante serviço de base comunitária, destacando-se por sua atuação nos campos de convivência e cultura (Silva et al., 2020). Com todas essas características, o CECCO torna-se um espaço de excelência também para a formação acadêmica, não como um laboratório de experimentação, mas como um local de vivência e profundo conhecimento social (Melo et al., 2016).

\section{Papo de Saúde}

O "PAPO DE SAÚDE" é um grupo de estudos no qual participam estudantes, professores e pesquisadores que tenham interesse em contribuir no contexto da educação em saúde, com populações que experimentam diferentes situações de vulnerabilidade. Tal fulcro permite que o trabalho do grupo se concentre em ouvir os indivíduos e as comunidades robustecendo o empoderamento no que se refere às suas necessidades no âmbito dos cuidados. Inicialmente, esteve associado ao Mestrado Profissional em Promoção de Saúde e à Pós-graduação em Saúde Pública de uma universidade particular da zona sul do município de São Paulo e contava com a participação de discentes e docentes dos referidos cursos. Na ocasião, as reuniões do grupo aconteciam semanal ou quinzenalmente, variando de acordo com os projetos em pauta. Dentre as pesquisas desenvolvidas pelo grupo, distinguem-se os seguintes trabalhos: o Facebook como meio de informação e cuidado, a elaboração de folder e cartilhas educativas, pesquisa sobre o autocuidado masculino na prevenção do câncer de próstata, perfil de homens que buscam serviços de saúde em área de vulnerabilidade social, a presença de acompanhante na consulta masculina e ações educativas no âmbito da saúde em espaços não convencionais. No momento, constitui-se em uma organização com outros laços institucionais, guardando o mesmo direcionamento.

Existe um vigoroso alinhamento entre o trabalho desenvolvido pelo grupo PAPO DE SAÚDE e o CENTRO DE CONVIVÊNCIA E COOPERATIVA (CECCO). Cabe ressaltar que, além desses aspectos, a proximidade do CECCO com o Centro Universitário, que sediava o PAPO DE SAÚDE, foi primordial para a realização do projeto. Estes aspectos sinalizam a relevância da inter-relação das atividades de pesquisa, extensão e ensino e os serviços.

Neste manuscrito, relata-se a experiência de uma RODA DE CONVERSA desenvolvida junto a um grupo de mulheres no mês de outubro de 2017, época da campanha de conscientização sobre o câncer de mama. Tratou-se de uma atividade contextualizada no âmbito da educação em saúde, embasada em ações primárias no combate à doença..

\section{Procedimentos Metodológicos}

Tratou-se de um estudo descritivo de caráter qualitativo, desenvolvido a partir de uma RODA DE CONVERSA (Melo et al., 2016). A escolha aconteceu por contribuir à integração ensino e serviço. A RODA DE CONVERSA oportuniza a relação 
entre profissionais de saúde, estudantes e usuários do serviço (Maropo et al., 2015). Ademais, favorece a relação dialógica entre os atores sociais envolvidos, contribuindo para que todos participem. Estes fatores reforçam a importância da Educação em Saúde como uma dinâmica voltada para a possibilidade de reflexão, compreensão e construção do conhecimento (Melo et al., 2016). Aspectos que compõem com a promoção da saúde, constructo vigoroso para a corresponsabilização do autocuidado. A configuração deste trabalho agrega a pesquisa-ação (Marques \& Vechiato, 2017), ferramenta metodológica que adota o pesquisador como membro ativo, juntamente com os participantes do estudo.

A concretização da ação foi implementada intersetorialmente por servidores do CECCO e integrantes do Grupo "PAPO DE SAÚDE". A implementação da experiência constituiu-se das seguintes etapas:

1- Discussão inicial entre os membros do Grupo PAPO DE SAÚDE (primeira e segunda semanas de agosto), quando se agregaram discentes e docentes da Pós-Graduação em Saúde Pública e Mestrado Profissional em Promoção da Saúde, que se interessaram em participar do desenvolvimento da ação (segunda semana do mês de agosto)

2- Reunião introdutória entre membros do grupo PAPO DE SAÚDE e trabalhadores do CECCO para a proposição da ação, a qual resultou na aprovação da proposta e definição dos papeis (terceira semana de agosto)

3- Reunião para levantamento de estudos e artigos abordando generalidades sobre o tema câncer de mama e autocuidado (quarta semana de agosto)

4- Elaboração de um pré-projeto (primeira semana de setembro)

5- Ajuste do projeto com a participação dos membros da equipe do PAPO DE SAÚDE e trabalhadores do CECCO (segunda e terceira semanas de setembro)

6- Distribuição de tarefas e adequação da agenda (primeira semana de outubro)

7- Divulgação do evento (segunda e terceira semanas de outubro)

8- Evento (quarta semana de outubro)

9- Avaliação do evento (primeira quinzena de novembro)

Para o direcionamento da conversa, foram feitas buscas sobre o câncer de mama nas seguintes plataformas: Biblioteca Virtual de Saúde (BVS), Literatura Latino-Americana e do Caribe em Ciências da Saúde (Lilacs), PubMed/Medline. A partir das leituras, foram discutidos os principais aspectos sobre a patologia, incluindo a existência e importância das ações primárias para o monitoramento da saúde da mama.

Argumentou-se sobre o ajuste de linguagem para facilitar o diálogo com a população na RODA DE CONVERSA.

Em função da natureza do serviço prestado pelo CECCO e por possíveis dificuldades dos frequentadores, optou-se por não identificar e numerar a população presente. Considerou-se, também, que ações ou gravações poderiam inibir a participação da população. Tal atitude foi discutida intersetorialmente e a decisão foi unânime.

Como cuidado adicional nesta primeira RODA DE CONVERSA e considerando a delicadeza do tema, optou-se pela participação exclusiva de mulheres como moderadoras e participantes.

As questões que nortearam a RODA DE CONVERSA foram:

1- Você conhece seu corpo (você se olha, você se toca)?

2- Você sabe o que é câncer de mama?

3- Você faz autoexame das mamas?

4- Você sabe como fazer o autoexame das mamas?

5- Conte se existem casos de câncer de mama na sua família

6- Qual grau de parentesco?

7- Você já procurou o serviço de saúde para algum cuidado da mama? 
O evento aconteceu em outubro de 2017, mês de conscientização sobre a prevenção e combate ao câncer de mama.

\section{Resultados}

A RODA DE CONVERSA confirmou-se como um método adjuvante à participação dos presentes, mesmo que algumas tivessem se colocado de maneira tímida. Realça-se que a RODA é constituída por meio de um diálogo, sem hierarquização ou categorização dos presentes; os moderadores abrem o diálogo e a expectativa é dar oportunidade de atuação (Silva et al., 2015).

Nesta experiência, foram apresentadas as vivências das participantes que, de forma inesperada, se adiantaram e encadearam os eixos temáticos que apareceriam nos momentos seguintes. Foi possível conhecer parte do "saber em saúde" do grupo, ocasião em que relataram as atitudes voltadas para a manutenção da saúde e preservação da vida, no âmbito do câncer da mama.

Abre-se um adendo para uma vez mais, pontuar que o cuidado de saúde mostra se, fortemente, associado com a questão de gênero e, frequentemente, é privilegiado pelas mulheres (Moraes, Costa, \& Silva, 2017). No início da RODA, o grupo de moderadoras abordou rapidamente a existência do câncer de mama entre homens, fato que, para alguns participantes, foi uma surpresa, desconhecimento que reforça a falta de informações sobre o assunto (Costa et al., 2020).

As participantes destacaram suas vivências intra e extrafamiliares relacionadas ao câncer de mama. Distinguiram o sofrimento relacionado ao tratamento, com ênfase à mastectomia e à mudança de aparência pela perda de cabelos causados pela quimioterapia. Ademais, citaram questões referentes à sexualidade das mulheres que adoeceram (Spada, \& Breda, 2017). Nesse momento, algumas participantes expressaram o medo da descoberta da doença, fator referenciado em estudo anterior (Bezerra, 2018). Houve relatos sobre acompanhamento de pacientes em sessões de tratamento e perdas de familiares e amigos, momento em que o grupo foi tomado por sentimentos de pesar.

Discutiu-se a relevância da hereditariedade no desenvolvimento da doença e a importância de monitoramento em mulheres consideradas vulneráveis, pela presença de casos na família (Gomes et al., 2020). Argumentou-se, também, que apesar da dificuldade de prevenção da doença, valoriza-se a eficiência do diagnóstico precoce (Cunha et al., 2018). A premissa apoia o conhecimento dos outros fatores de risco como: etilismo, tabagismo, sedentarismo, sobrepeso e questões hormonais no pós menopausa (Agostinho, Lima, Ferreira, 2019). A exposição fez emergir asserções relacionadas à vida saudável e ações primária de autocuidado em todos os momentos e não somente no mês que destaca o câncer da mama.

No que se refere ao autocuidado, as participantes ressaltaram as tarefas excessivas na rotina da vida como um dos empecilhos para que a rotina não aconteça. Mencionaram a falta de tempo como vilão dos cuidados, pois disponibilizam seus esforços para a atenção com casa, filhos e, em alguns casos, trabalho (Oliveira \& Martins, 2018). O conhecimento do corpo, por meio de observação, uso de espelho ou toque não integram o cotidiano do grupo. As afirmações deram margem para ressaltar a importância do cuidado de quem cuida. Observou-se que tais observações aproximaram as monitoras e as frequentadoras do CECCO, já que a questão repercutiu em todo grupo.

As participantes expuseram, de forma contundente, as dificuldades de acesso aos serviços e a demora no que se refere aos procedimentos e resultados de exames (Prado, Loiola, Guimarães, Ohara, Oliveira, 2020). Essas observações estão consonantes com o que se observa no Sistema Único de Saúde (SUS), que por ser um serviço que responde pela saúde da população, apresenta limites no atendimento.

Dentre os efeitos produzidos pala experiência, sublinha-se o impacto da RODA DE CONVERSA nos discentes do grupo PAPO DE SAÚDE. Usualmente, a formação concentra-se na força do conhecimento acadêmico e, na maior parte do tempo, são raros os momentos de oitiva da população/comunidade. Os docentes tiveram a oportunidade de observar a atuação de seus alunos, o que foi inovador para o grupo. Para os servidores do CECCO, foi o momento de observar o impacto e 
influência do trabalho no desempenho e, porque não dizer, no protagonismo das frequentadoras. Foi relevante conhecer as vivências das participantes e seus conhecimentos sobre o tema.

Ao final da RODA DE CONVERSA, foram distribuídos folders que tratavam sobre generalidades sobre o câncer de mama.

O evento foi considerado um evento bem sucedido e, diante disso, foram acordadas possíveis futuras novas RODAS DE CONVERSA alicerçadas, principalmente, nas necessidades e interesse do público alvo.

\section{Considerações Finais}

O diálogo é um disseminador de informações e a RODA DE CONVERSA é uma potente ferramenta para tal.

Um número reduzido de participantes mostrou conhecimento sobre o câncer de mama masculino, fato que aponta para a necessidade de disseminação sobre o tema.

As participantes destacaram a necessidade de cuidados em todas as épocas do ano e não apenas no mês de outubro, instituído como o momento de conscientização e combate ao câncer de mama.

Ainda, as mulheres que frequentam o CECCO manifestaram familiaridade com o tema câncer de mama feminino. Pontuaram suas vivências, evidenciando o medo do diagnóstico e dos efeitos do tratamento, com ênfase para a mastectomia e a perda de cabelos. O temor do diagnóstico faz com que parte das mulheres do grupo não se auto examine. Um número expressivo entre as participantes culpou a falta de tempo para não praticarem o autocuidado, aspecto surpreendentemente concordante com algumas monitoras.

Foram mencionadas as dificuldades com os serviços de saúde: acesso aos procedimentos e demora nos resultados.

Chamou atenção o protagonismo das participantes, já que as monitoras realmente só auxiliaram na discussão das conversas.

O evento trouxe várias contribuições: a vivência das participantes; o conhecimento do impacto do trabalho de CECCO na vida das mulheres que frequentam o serviço; e no que diz respeito ao grupo PAPO DE SAÚDE, a observação dos docentes quanto à atuação dos alunos e à observação de trabalho de promoção e educação em espaços não convencionais.

A tarefa mostrou-se promissora e, suporte para novos encontros que dependerão da disponibilidade das equipes e, seleção de temas compatíveis com os interesse e as necessidades do público em pauta.

A avaliação pontuou o êxito da iniciativa e a perspectiva de futuras RODAS DE CONVERSA, que poderão se consolidar como uma atividade mais frequente entre CECCO e PAPO DE SAÚDE.

\section{Referências}

Agostinho, J. C., Lima, T. V., \& Ferreira, R. C. V. (2019). Análise dos fatores de risco do Câncer de Mama e avaliação da campanha preventiva "Outubro Rosa”. Revista Saúde UniToledo, 3 (2), 97-108.

Aleixo, J. M. P., \& Araújo Lima, E. M. F. (2017). Invenção e produção de encontros no território da diversidade: cartografia de um Centro de Convivência/Invention and production of encounters on diversity's territory: cartography of a Community Center. Cadernos Brasileiros de Terapia Ocupacional, 25 (3), 649-659.

Alvarez, A. P. E., Silva, J. O., \& Oliveira, A. C. M. (2016). Centro de Convivência e Cultura: diálogos sobre autonomia e convivência. ECOS - Estudos Contemporâneos da Subjetividade, 6 (1), 5-19.

Alves, P. C., Ferreira, I. S., Santos, M. C. L., Almeida, A. O. A. D., \& Fernandes, A. F. C. (2019). Efeitos de intervenção educativa no conhecimento e atitude sobre detecção precoce do câncer de mama. Revista Rene (Online), 20 (1).

Ayala, A. L. M., Anjos, J. C. D., Cassol, G. A., \& Höfelmann, D. A. Sobrevida em 10 anos em mulheres com câncer de mama: coorte história de $2000-2014$. (2019). Ciência \& Saúde Coletiva, 24 (4), 1537-1550.

Bankoff, A. D. P. (2014). Qualidade de vida de mulheres que realizaram cirurgias de mama. Conexões, 12 (1), 42-58.

Barata, R. B. (2009). Como e por que as desigualdades sociais fazem mal à saúde. Editora Fiocruz. 
Bezerra, T. R. M. (2018). Conhecimento e práticas de autocuidado para detecção precoce do câncer de mama entre mulheres com fator de risco familiar. Trabalho de Conclusão de Curso (Bacharelado em Enfermagem), Universidade Federal de Campina Grande, Cajazeiras, PB, Brasil.

Caçapava, J. R., Colvero, L. A., \& Pereira, I. M. T. B. (2009) A interface entre as políticas públicas de saúde mental e promoção da saúde. Saúde e Sociedade, $18(3), 446-455$

Costa, G. O. P., Moura, J. C., Ribeiro, A. M. N., Vaz, B. A., Silva Santana, R., Baldoino, L. S., \& Pinto, N. V. R. (2020). Nem todo outubro é rosa: homem também pode ter câncer de mama. Um relato de experiência. Research, Society and Development, 9 (11).

Costa, P. V. D. P., Silva, J. M. L., Silva, L. C., Rocha, S. G., Costa, L. F., Soares, M. K. M., \& Tavares, I. I. S. (2020). A educação em saúde como ferramenta no combate ao câncer de mama: relato de experiência. Research, Society and Development, 9 (10).

Cunha, A. R., Almeida, A. A., Oliveira, S. P. S., Paulino, T. S. C., Junior, L. S. D. S., \& Fontinele, D. C. S. D. S. (2018). O papel do enfermeiro na orientação, promoção e prevenção do câncer de mama. Revista Humano Ser, 3 (1), 160 - 173.

Gomes, K. A., Monteiro, L. N., Oliveira, M. E. C., Nóbrega, W. F. S., Mota, G. B. C., Barbosa, D. V., \& Melo Júnior, S. A. (2020). Conhecimento de usuárias de um serviço público de saúde sobre fatores de risco e de proteção para o câncer de mama. Research, Society and Development, 9 (9), e498997521e498997521.

Maropo, J. M. B., Santana, D. P., Oliveira Filho, V. F., Almeida, C. V. B., \& Araújo, I. M. (2015). Saúde da mulher na roda: projeto de extensão dialoga sobre prevenção na comunidade. Revista de Ciências da Saúde Nova Esperança, 3 (2), 106-112.

Marques, C. A. G., \& Vechiato, F. L. (2017). Interoperabilidade entre Repositório Institucional e Sistema Integrado de Gestão de Atividades Acadêmicas. Informação \& Tecnologia (ITEC), 4 (2), 4-25.

Martins, I. R., Soares, J. S., Santos, J. P. A., Silva, T. K. R., Do Sacramento, H. T., Pampolim, G., \& Sogame, L. C. M. Valorização do contexto sociocultural na criação do vínculo entre uma mulher com HPV e um serviço de saúde. (2018). Cadernos de Educação, Saúde e Fisioterapia, 5 (10).

Melo, R. H. V. D., Felipe, M. C. P., Cunha, A. T. R. D., Vilar, R. L. A. D., Pereira, E. J. D. S., Carneiro, N. E. A., \& Diniz Júnior, J. (2016). Roda de conversa: uma articulação solidária entre ensino, serviço e comunidade. Revista Brasileira de Educação Médica, 40 (2), $301-309$.

Moraes, M. C. L. Promoção da saúde: visitando conceitos e ideias. (2017). Revista Família, Ciclos de Vida e Saúde no Contexto Social, 5 (1), $75-79$.

Moraes, M. C. L., Oliveira, R. C., \& Silva, M. J. (2017). Uma questão masculina: conhecendo possíveis entraves para a realização dos exames de detecção do câncer de próstata. Revista Medica Herediana, 28 (4), 230-235.

Oliveira, A. S. (2018). O papel do enfermeiro na assistência ao pré-natal de baixo risco. Monografia de Pós Graduação, Universidade da Integração Internacional da Lusofonia Afro-brasileira, São Francisco do Conde, BA, Brasil.

Oliveira, B. B., \& Martins, I. B. D. S. (2018). Câncer de mama e de colo de útero: conhecimento, percepção e atitude de trabalhadoras de centro de ensino superior. Artigo de Conclusão de Curso, Centro Universitário de Maringá, Maringá, PR, Brasil.

Prado, N., Loiola, P., Guimarães, T., Ohara, E. C. C., \& Oliveira, L. D. R. (2020) Gestante com diagnóstico de câncer de mama: prevenção, diagnóstico e assistência. Brazilian Journal of Health Review, 3 (1), 1109-1131.

Raimondi, G. A., Paulino, D. B., Mendes Neto, J. D. P. M., Diniz, L. F., Rosa, G. F., Limirio Juniori, V. L., \& Leonardi, C. B. G. (2018). Intersetorialidade e Educação Popular em Saúde: no SUS com as Escolas e nas Escolas com o SUS. Revista Brasileira de educação médica, 42 (2), $73-78$.

Rodrigues, J. D., Cruz, M. S., \& Paixão, A. N. Uma análise da prevenção do câncer de mama no Brasil. (2015). Ciência \& saúde coletiva, 20 (10), $3163-3176$.

Rodrigues, T. B., Stavola, B. D., Bustamante-Teixeira, M. T., Guerra, M. R., Nogueira, M. C., Fayer, V. A., \& Santos-Silva, I. (2019). Sobrerrastreio mamográfico: avaliação a partir de bases identificadas do Sistema de Informação do Câncer de Mama (SISMAMA). Cadernos de Saúde Pública, 35 (1), e00049718.

Silva, A. P. G., Barbosa, C. L., \& Bonini-Trenche, M. C. (2020). Atuação de fonoaudiólogos em Centros de Convivência e Cooperativa (CECCO): trajetórias e desafios da formação profissional. Distúrbios da Comunicação, 32 (1), 26-40.

Silva, J. V., Domingues, E. A. R. (2017). Adaptação cultural e validação da escala para avaliar as capacidades de autocuidado. Arquivos de Ciências da Saúde, $24(4), 30-36$

Silva, F. M. O., Maia, G. M. C., Rocha, I. S., \& de Lima Carvalho, C. M. (2015) "Roda de conversa na promoção da saúde física e mental de mulheres. Saúde em Foco: Temas Contemporâneos, 3, 603-614.

Spada, B. L., Breda, P. (2017). Breve análise do autocuidado em relação a saúde da mulher no município de Pinhalzinho-SC. Anuário Pesquisa e Extensão Unoesc São Miguel do Oeste, 2, e13008.

Tavares, V. M. C., Angelo Neto, L. M., Pereira, E. S., Taveira, M. D. G. M. M., Cavalcante, J. K., \& Correia, D. S. (2020). Roda de conversa: atenção integral à saúde das mulheres e questões de gênero. Brazilian Journal of Development, 6 (8), 61501-61510.

Viegas, A. C., Muniz, R. M., Cardoso, D. H., Santos, B. P., Machado, J. B., \& Lindemann, L. G. (2019). Prevenção do câncer de mama: conhecimento de mulheres de uma unidade saúde da família. Saúde em Revista, 19 (51), 57-76. 